\title{
Luminosity Function and Evolution of Optically Selected QSOs
}

\author{
Lutz Wisotzki \\ Universität Potsdam, Am Neuen Palais 10, D-14469 Potsdam, Germany
}

\begin{abstract}
I summarise a few recent results on the evolution of optically selected QSOs, with special emphasis on the notoriously difficult but physically important extremes at low and high luminosities and redshifts. It seems that quasar evolution is a more complex phenomenon than has often been assumed, and the new generation of surveys is just about to make this visible.
\end{abstract}

\section{Introduction}

Luminosity functions are an important tool to study the population properties of Active Galactic Nulcei. The strong evolution of nuclear activity in galaxies over cosmic epochs is most evidently visible in the changing luminosity function, implying that the space density of present-day luminous AGN is only $\sim 10^{-3}$ of that of high-redshift quasars. Precise quantitative measurements of the AGN luminosity function, however, have proven to be surprisingly difficult. Comparing the quasar $L F$ at different redshifts inevitably requires the usage of different surveys, each with its own selection criteria and ideosyncrasies. Especially at extremely high and low luminosities and redshifts, statistical coverage is still poor, even though current surveys are now churning out new quasars by the thousands.

A good knowledge of the AGN luminosity function is also a prerequisite to understand the thermal history of the early universe. QSOs provide a significant fraction of the metagalactic UV radiation field that keep the intergalactic medium ionised, yet current estimates are highly uncertain due to the poorly constrained contribution of low-luminosity AGN.

\section{The Optical QSO Luminosity Function}

\subsection{General Remarks}

Quasars are still most easily found at optical wavelengths, due to the existence of large-format detectors and the fact that optical spectra are required to get redshifts. Optical QSO surveys have therefore been leading in shaping the overall picture of the QSO luminosity function (QLF) and its evolution. Particularly influential was the work of Boyle and collaborators (Boyle et al. (1988)) who argued that the shape of the QLF remains invariant with redshift, and evolution manifests itself as a mere shift in characteristic luminosity (Pure Luminosity 


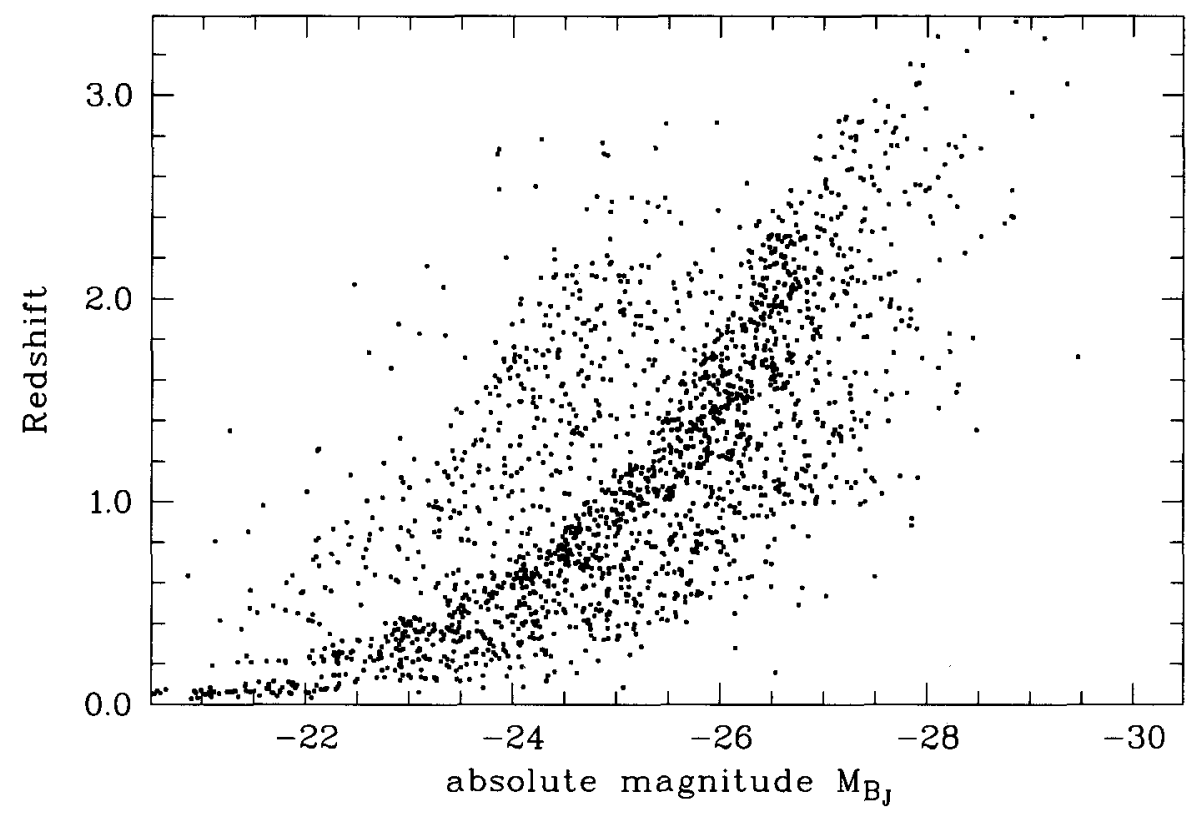

Figure 1. Distribution of redshifts and absolute blue magnitudes for the combined sample.

Evolution, PLE). PLE characteristics have also been found for AGN evolution at X-ray (Jones et al. (1997)) and radio wavelengths (Dunlop \& Peacock (1990)), albeit with much poorer statistics than in the optical.

More recently, the applicability of simple PLE models have been questioned based on new survey data. Hewett et al. (1993) showed that the PLE model predicts too few bright and too many faint low-redshift $(z \lesssim 1)$ quasars. This fact has been independently confirmed by our Hamburg/ESO survey results (Köhler et al. (1997); Wisotzki (2000a); cf. also Reimers \& Wisotzki, these proceedings), where the mismatch between PLE predictions and actual data is highly significant. It can be safely said PLE as a global description of quasar evolution is ruled out because the QLF changes its shape with cosmic time.

\subsection{Semi-Parametric Analysis}

We have recently conducted a new attempt to model the evolving QLF, working on a merged sample of six optical QSO surveys. Altogether, the sample contains 1946 objects, and coverage of the Hubble diagram is more or less complete for $M_{B_{J}}<-23$ and $z<3$, although the scarcity of low-luminosity AGN at high redshifts and of high-luminosity AGN at low redshifts is still prominent (Fig. 1). We do not consider for the moment the very high redshift range, for which the data are even scarcer, although much progress is currently made (see also below).

When modelling the QLF, one is confronted with the choice between nonparametric and parametric estimators. The former has the virtue of being free 
of premeditated assumptions, but is unfortunately prone to a number of numerical biases such as evolution within redshift bins (see also Petrosian, these proceedings). Parametric model fitting, on the other hand, avoids these biases but may be too inflexible to describe the actual data (especially when small samples demand a small number of parameters). To overcome these limitations, we have devised a hybrid 'semi-parametric' scheme (Wisotzki (1998)), featuring the following properties: (1) The QLF is described as polynomial in $\mu \propto \log L$. (2) Evolution is parameterised as a polynomial in the variable $\zeta=\zeta(z)$. (3) The general expression for an evolving QLF is a bivariate polynomial of $\mu$ and $\zeta$,

$$
\log \phi=\sum_{i=0}^{n} \sum_{j=0}^{m} C_{i j} \mu^{i} \zeta^{j}
$$

This 'free form' LF representation was introduced by Peacock \& Gull (1981) for the analysis of radio surveys, but has rarely been used otherwise. Its main advantage is that given samples of sufficient size, almost any QLF shape can be recovered by fitting the above form to the data. The result is a QLF estimate that is an unbinned continuous function, yet free of the above mentioned biases. Notice that the fitting parameters in this method are not interesting in themselves and basically meaningless in physical terms, especially for a high-order QLF (this is why the method could be called 'semi-parametric').

Applying the method to our combined sample, we derive the following constraints (see also Fig. 2): (1) No global model with an invariant QLF is acceptable - both PLE as well as its counterpart PDE (Pure Density Evolution) are ruled out with high significance. (2) An acceptable fit is achieved only with a high-order luminosity-dependent density evolution scheme. (3) The evolution rates depend both on luminosity and on redshift. (4) Evolution at $z<1$ is much faster than at $z>1$. (5) There is no evidence for significant slow-down of evolution around $z \approx 2$ as suggested by earlier analyses and also required in modelling the evolution of X-ray selected AGN.

\subsection{Evolution in Cosmological Epochs}

The fact that a global fit to our $0<z<3$ sample is only achieved by employing a complicated QLF model and many free parameters indicates that the underlying physical processes have not been isolated. It is therefore interesting to find that at least for our current sample the description becomes very simple if the sample is split into low- and high-redshift subsets, each containing roughly $50 \%$ of the sample.

'High' Redshift $(1 \lesssim z \lesssim 3)$ : The QLF shows strong curvature with a steep high-luminosity and a shallow low-luminosity tail. The shape is wellapproximated by a 3rd-order polynomial in $\log \phi(M)$ (in fact better than the with the canonic 'double power law') and is nearly invariant within the covered redshift range. Surprisingly, both PLE and PDE are equally acceptable. While 20 free-form coefficients were not enough for the global description, a simple 5 -parameter model is perfectly adequate for half the sample. This explains why the PLE model performed so well in the past and was still favoured in the recent analysis by Boyle et al. (2000): These samples are dominated by intermediateredshift QSOs (e.g., $>70 \%$ of the AAT sample are at $1<z<2.2$ ), and for 


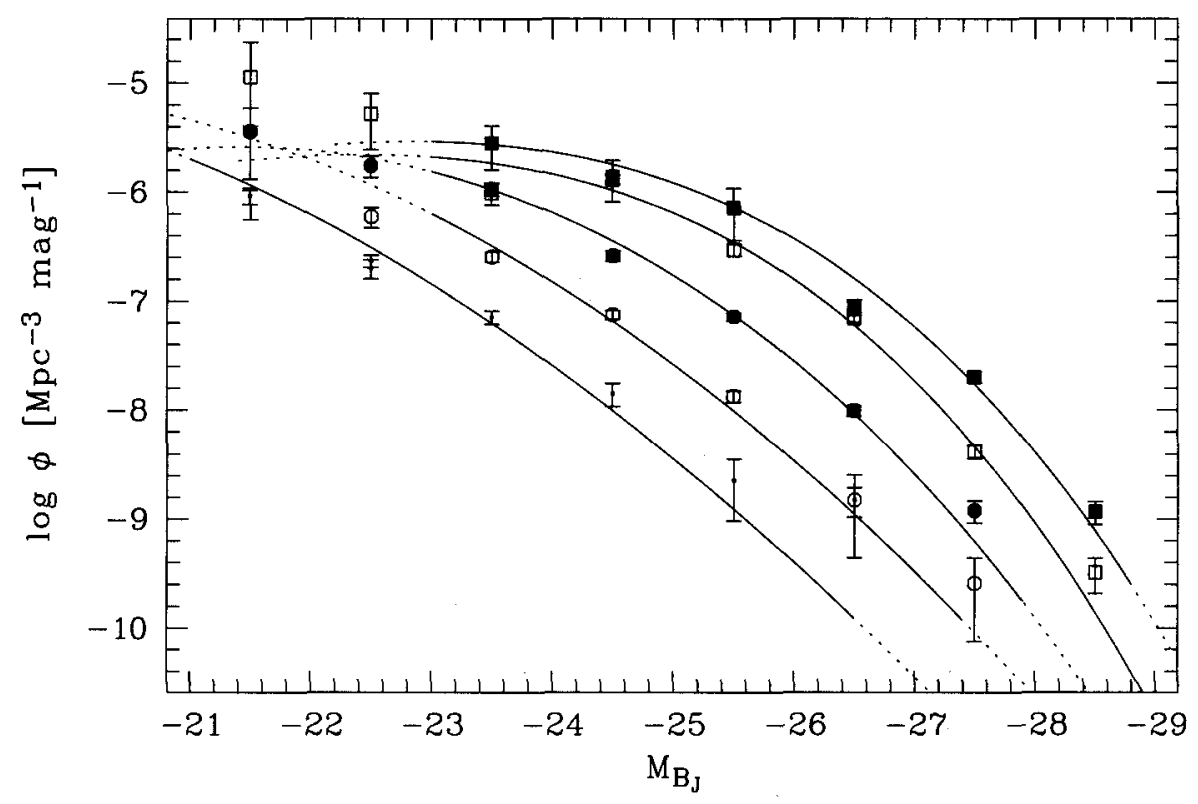

Figure 2. Semiparametric estimate of the evolving QLF in five redshift shells $(z<0.3,0.3<z<0.7,0.7<z<1.25,1.25<z<2$, $2<z<2.9)$.

these redshifts PLE indeed is an acceptable description (although PDE does just as well).

Low Redshift $(z \lesssim 1$ ): The low-redshift QLF is almost a single power law, with very little curvature at least within the classical QSO domain. If the sample is restricted in absolute magnitude to $M_{B_{J}} \lesssim-23$, an excellent description is achieved with pure density evolution, while PLE performs much poorer. This shows an interesting parallel to the results of Miyaji et al. (2000) who found that PDE was almost a good fit to their X-ray sample and failed only because of a slight overproduction of $z>1$ AGN.

\section{High-Luminosity AGN at High Redshifts}

General wisdom says that the peak of the QSO space density lies between $z \simeq 2$ and $z \simeq 3$. However, if quasar luminosities depend in some way on the masses of their underlying host galaxies, the location of the actual maximum may well depend on the luminosity range sampled. There is some evidence that this is indeed the case, in the sense that the peak is shifted towards higher redshifts for the highest luminosity QSOs. If confirmed, this would rule out at least the naive version of a hierarchical model with QSO luminosities being proportional to the corresponding dark matter haloes. 


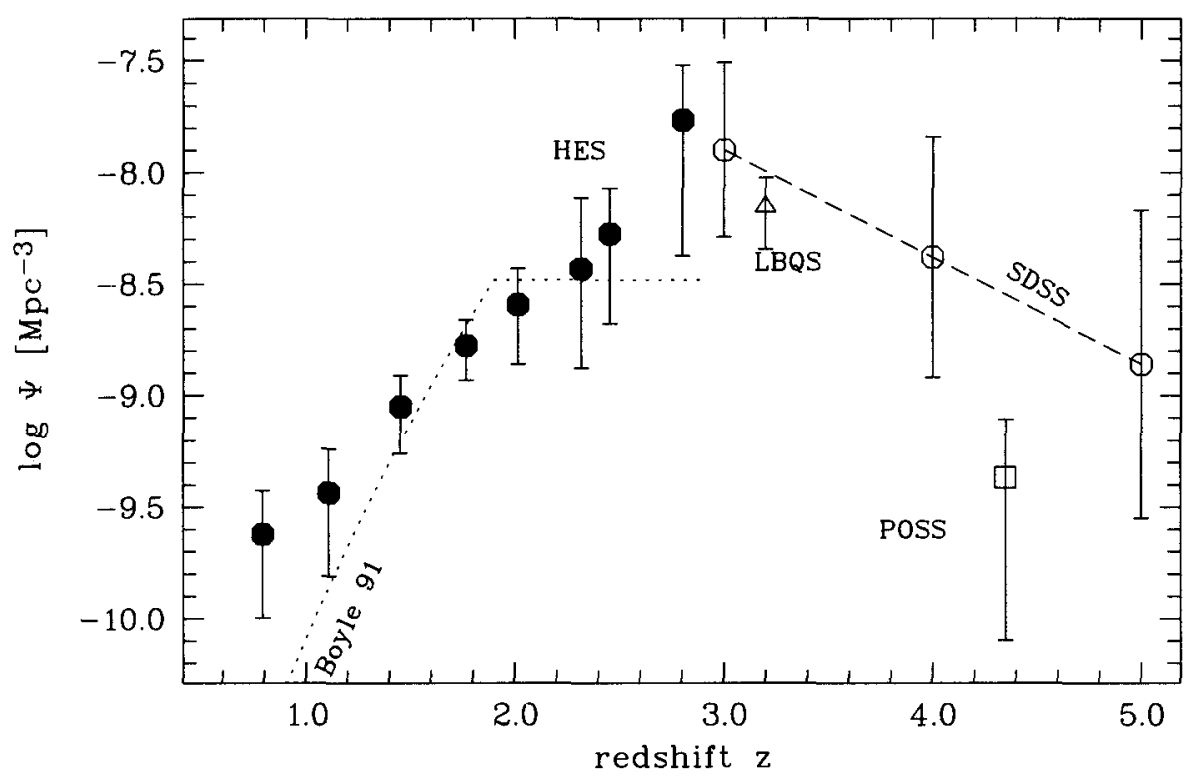

Figure 3. Evolution of the space densities of the most luminous QSOs. The SDSS points have been constructed from the parametric model by Fan et al. (2001); the error bars include the extrapolation of that model to $M_{B}=-28$.

Unfortunately, the maximum itself has so far been escaped detection. The main constraint for extremely luminous QSOs with $M_{B}<-28$ at $z<3$ comes from the Hamburg/ESO survey (Wisotzki (2000a)) from where it appears that their evolution proceeds at almost constant rate until $z \simeq 3$ (Fig. 3), with no indication of a slow-down. At even higher redshifts, the results were quite contentious until recently the first results from SDSS (Fan et al. (2001)) demonstrated that the decline towards $z=5$ is definitely real. However, Fan et al. also found that the high-redshift high-luminosity tail of the QLF is much flatter than at $z \simeq 2-$ this is equivalent to saying that the evolution rate depends on luminosity. Accordingly, very high-luminosity QSOs have a slower decline, or alternatively they may have a maximum at higher $z$. Figure 3 shows that for $M_{B}<-28$, the SDSS and HES results are not in conflict, as they nicely bracket the probable maximum. It is nevertheless puzzling to realise that this peak corresponds to a cosmic time span of less than 1 Gyr FWHM.

Finally, one additional source of uncertainty should be mentioned: in order to combine low- and high-redshift space densities, the spectral energy distributions need to be known; usually they are approximated as power laws $f_{\nu} \propto \nu^{\alpha}$ with $\alpha=-0.5$ or by composite QSO spectra. Such approximations are a nonnegligible source of uncertainty, and past specifications have even lead to a systematic overestimate of high-redshift QSO luminosities by up to $0.5 \mathrm{mag}$ at $z \simeq 2$ (Wisotzki (2000b)). Consequently, the inferred evolution rates are signifi- 
cantly reduced and now in fact compatible with what is found in X-ray surveys. Much more work needs to be done on this aspect in the future.

\section{High-Luminosity AGN in the Local Universe}

Before discussing the results, one important operational caveat should be mentioned that has mostly been neglected: Source photometry of low-redshift AGN using standard techniques will inevitably lead to significant host galaxy contributions, even in the $B$ band, even for high-luminosity AGN. In the HES, all flux measurements are essentially nuclear rather than total magnitudes, thereby largly eliminating host galaxy bias, but other low-redshift samples are less well defined in terms of their AGN luminosities. Comparing the resulting surface and space densities is therefore difficult and should be regarded with suspicion unless host galaxy contributions are explicitly accounted for.

Qualitatively it is now clear that the bright end of the QLF flattens towards low redshifts; this is confirmed by several surveys (Goldschmidt \& Miller (1998); Wisotzki (2000a); Grazian et al. (2000); see Fig. 2). In the same manner as above for the SDSS results, this implies a reduced evolution rate for most luminous QSOs. Physical origins for this effect are not yet known. It has been speculated that radio-quiet and radio-loud populations might evolve with different rates, which would explain the very high fraction of RLQs in the Palomar/Green Bright Quasar Survey (Kellerman et al. (1989)). However, our ongoing radio follow-up of the Hamburg/ESO survey shows that the RLQ fraction in the HES is $\sim 10$ $15 \%$ even for high-luminosity AGN at low redshifts, in stark contrast to the BQS. Furthermore, RQQ and RLQ have indistinguishable redshift distributions. The conclusion so far is that the radio-loud population cannot be responsible for the flattening of the QLF.

\section{Low-Luminosity AGN in the Local Universe}

Although an abundant species, an accurate assessment of the contribution of lowluminosity AGN to the QLF is fraught with technical difficulties, mainly arising from the fact that $L_{\text {host }} \gtrless L_{n u c}$ : How to disentangle nuclear and host luminosity contributions? How to define limiting survey magnitudes? How to combine different surveys? Several heroic attempts in the past have tried to account for these problems at least in a statistical manner (e.g., Cheng et al. (1985); Marshall (1987)), but mostly these were lacking adequate data. Recall also that magnitudes from galaxy surveys are meaningless when used to construct a nuclear LF (e.g., the Sy 1 galaxy LF derived in the CfA survey, Huchra \& Burg (1992) is mostly a host galaxy luminosity function).

In the last years, a few authors have estimates the local LF of 'Type 1' AGN taking care of these problems. First, the local QLF from the Hamburg/ESO survey (Köhler et al. (1997)) included individual host subtraction. The LF was found to be close to a single power law down to $M_{B} \simeq-18$, but the statistics at low luminosities were very poor. More recently, the optical LF of low-redshift X-ray-selected AGN was constructed by Londish et al. (2000) using HST imaging and proper subtraction of host galaxy contributions. The 


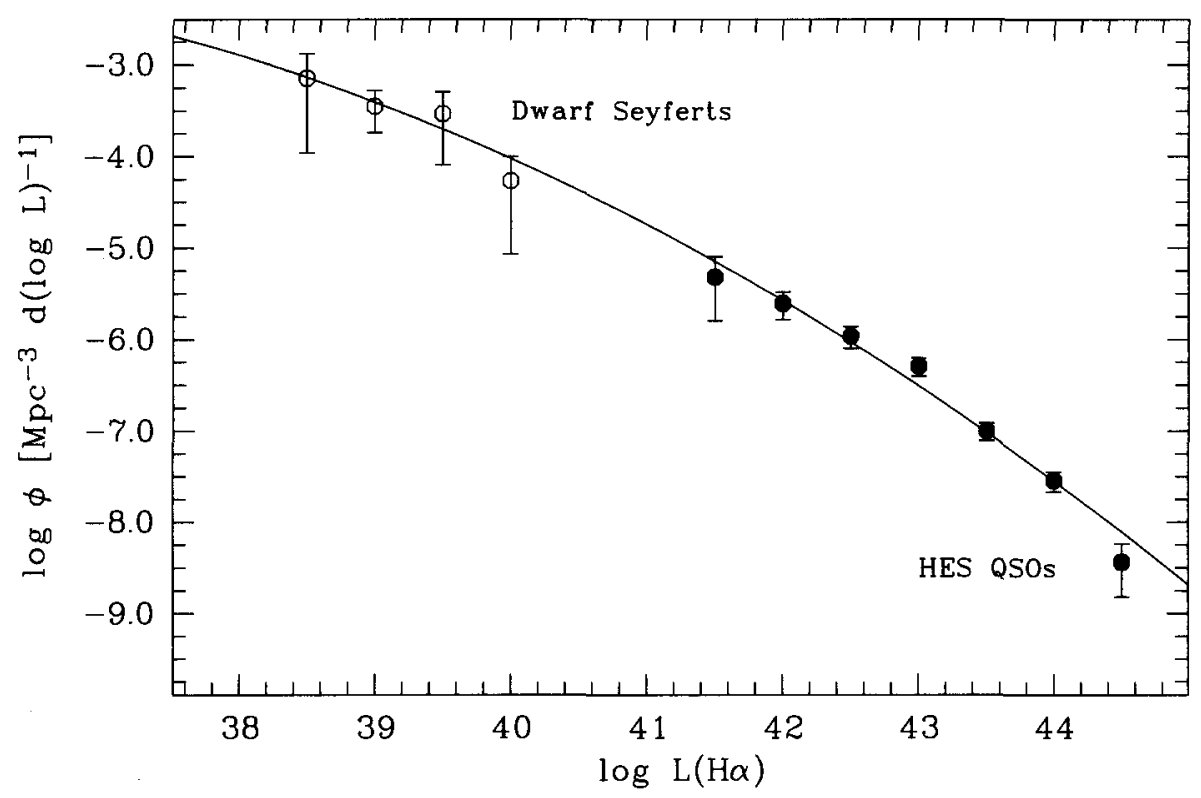

Figure 4. $\mathrm{H} \alpha$ luminosity function of low-redshift AGN. Note the smooth connection between high-luminosity quasars and lowluminosity Seyferts. The solid line is a free-form polynomial fit to the data.

power law slope for $M_{B} \lesssim-20$ was confirmed, but there is a significant turnover towards low luminosities.

The Ho Luminosity Function of Local AGN It is known that the $\mathrm{H} \alpha$ line flux is well correlated with blue continuum magnitude. Furthermore, it is physically expected to reflect the number of UV photons intercepted by the BLR. We adopt therefore the integrated luminosity of the broad component of $\mathrm{H} \alpha$ as an alternative measure of nuclear power in type 1 AGN. Its importance lies in the fact that this quantity can be separated spectroscopically from the host galaxy, which is relatively easy to accomplish in all except the most feeble active nuclei.

For spectroscopically complete AGN samples it is then straightforward to construct an $\mathrm{H} \alpha$ luminosity function of low-redshift AGN. We have done this for two samples: (1) Our own HES dataset, using the spectra from the candidate conformation observations. (2) The 'Dwarf Seyfert' sample of Ho et al. (1995), using the deblended broad $\mathrm{H} \alpha$ luminosities from $\mathrm{Ho}$ et al. (1997). The combined LF is shown in Fig. 4. At brighter luminosities, the shape of the $\mathrm{H} \alpha \mathrm{LF}$ is basically indistiguishable from the low- $z$ QLF in the broad $B_{J}$ band (Wisotzki (2000a)), in particular there is no evidence in the HES data for a turnover into a flatter low-luminosity tail. This is dramatically confirmed by the Dwarf Seyferts forming a seamless continuation, only slightly below a simple power law extrapolation from intermediate towards low luminosities. 
At face value, this seems to be in strong conflict with the results of Londish et al. (2000), assuming that both soft X-rays and broad $\mathrm{H} \alpha$ luminosities represent similar primary emission components - essentially, both are used as estimators of the UV output. That latter assumption may be incorrect if the 'Dwarf Seyferts' are operating on ADAF-type accretion flows as suggested by Ho (1999); (also these proceedings). Furthermore, it is by no means obvious that X-ray selected AGN should yield the same optical LF as an optically selected sample.

\section{Low-Luminosity AGN at High Redshifts}

The space density of high- $z$ low-luminosity AGN is still very poorly known, at $z \gtrless 3$ even almost unconstrained. However, this quantity is extremely important also beyond AGN physics, as the AGN contribution to the metagalactic ionising UV background radiation field depends sensitively on the shape of the lowluminosity end of the QLF. Useful samples are small, and spectroscopic follow-up is expensive. The faintest currently available complete samples are:

- Marano field (Zitelli et al. 1992): 52 AGN, z $<2.9$ and B $<22$. Partly visual selection.

- CFRS (Schade et al. 1996): 6 AGN with $\mathrm{z}<3$ and I $<22.5$; no QSOspecific selection.

- HDF (Jarvis \& MacAlpine 1998; Conti et al. 1999; Sarajedini et al. 2000): Between 1 and 20 AGN candidates, but only 1 certain spectroscopic identification (of a type 2 AGN).

Most researchers simply assume that the faint-end slope derived at lower $z$ can be extrapolated; given the presently existing data, it is futile to speculate about stringent tests of such assumptions. This sobering situation is hopefully to change over the next few years with more powerful multiplex spectroscopic facilities arriving at large telescopes. The new generation of wide-field imagers, hase made generating faint candidate samples a possibility, but spectroscopic follow-up is still the major hurdle. A possible approach to circumvent this bottleneck is to use photometric redshifts based on a multicolour survey database. To take an example, our recently started COMBO-17 survey (Wolf et al. (2001)) features deep images in 17 different photometric bands ( 5 broad- and 12 mediumband filters) and can yield redshift estimates with an accuracy fully sufficient for luminosity calculations. First results will be available shortly.

\section{Conclusions}

To a very qualitative level, the general picture of quasar evolution is no longer contentious, but several quantitative issues are still unsolved. We can probably say that the uncertainties at intermediate redshifts and intermediate luminosities are relatively small, and that the overall evolution properties of such objects can be considered known, but that the more extreme objects maintain their elusive status. Some of the central questions that are still open: 
- When was the peak of QSO activity in the universe? Does its location or shape depend on luminosity, and if so, in what way? Does it make sense, after all, to speak of a well-defined 'quasar epoch', or can substantial AGN formation be traced out to very high redshifts?

- The interpretation of traditional single-band surveys hinges on the assumption of average spectral energy distributions. New multicolour surveys can measure individual SEDs and vastly reduce the $K$ correction uncertainties, but combining low- and high- $z$ surveys remains a problem. Which 'luminosity' is sought to be represented?

- The local AGN distribution properties are still very uncertain and difficult to disentangle from their host galaxies. If indeed ADAF-type solutions become important at very low $L$, then how does the transition regime manifest itself in the luminosity function? Intermediate luminosity scales need to be probed - here the new large galaxy surveys such as 2dFGRS, SDSS and VIMOS will yield important constraints.

The fundamental question of 'obscured AGN' has not been addressed here, as optical selection is known to systematically avoid such objects. There can be no doubt that a significant fraction of low-luminosity (Seyfert-type) AGN is removed from directly seeing the central engine through obscuration. On the other hand, it is not clear and still a matter of debate whether this is an important effect also for high-luminosity AGN, as their much stronger incident UV radiation fields and possibly also winds might have a clearing effect on any present obscuration screens. All these issues will be taken up by other contributors to these proceedings. As a final remark on the continuing usefulness of optical AGN surveys, let me note that the whole issue of what AGN fraction might be obscured is irrelevant for the cosmologically important task of estimating the AGN contribution to the metagalactic UV background; here the statement holds: What You See Is What You Get.

\section{References}

Boyle B. J., Shanks T., Croom S. M., Smith R. J., Miller L., Loaring N., Heymans C., 2000, MNRAS, 317, 1014

Boyle B. J., Shanks T., Peterson B. A., 1988, MNRAS, 235, 935

Cheng F.-z., Danese L., de Zotti G., Franceschini A., 1985, MNRAS, 212, 857

Dunlop J. S., Peacock J. A., 1990, MNRAS, 247, 19

Fan X., Strauss M. A., Schneider D. P., et al., 2001, AJ, 121, 54

Goldschmidt P., Miller L., 1998, MNRAS, 293, 107

Grazian A., Cristiani S., D’Odorico V., Omizzolo A., Pizzella A., 2000, AJ, 119, 2540

Hewett P. C., Foltz C. B., Chaffee F. H., 1993, ApJ, 406, L43

Ho L. C., 1999, ApJ, 516, 672

Ho L. C., Filippenko A. V., Sargent W. L., 1995, ApJS, 98, 477

Ho L. C., Filippenko A. V., Sargent W. L., Peng C. Y. T., 1997, ApJS, 112, 391

Huchra J., Burg R., 1992, ApJ, 393, 90 
Jones L. R., McHardy I. M., Merryfield M. R., et al., 1997, MNRAS, 285, 547 Kellerman K. I., Sramek R., Schmidt M., Shaffer D. B., Green R., 1989, AJ, 98, 1195

Köhler T., Groote D., Reimers D., Wisotzki L., 1997, A\&A, 325, 502

Londish D., Boyle B. J., Schade D. J., 2000, MNRAS, 318, 411

Marshall H. L., 1987, AJ, 94, 628

Miyaji T., Hasinger G. ., Schmidt M., 2000, A\&A, 353, 25

Peacock J. A., Gull S. F., 1981, MNRAS, 196, 611

Wisotzki L., 1998, AN, 319, 257

Wisotzki L., 2000a, A\&A, 353, 853

Wisotzki L., 2000b, A\&A, 353, 861

Wolf C., Dye S., Kleinheinrich M., Meisenheimer K., Rix H.-W., Wisotzki L., 2001, A\&A, 377, 442 